\title{
Short Paper: Practically Unbounded One-Way Chains for Authentication with Backward Secrecy*
}

\author{
Roberto Di Pietro Antonio Durante Luigi Mancini Vishwas Patil \\ Dipartimento di Informatica, Università di Roma "La Sapienza" \\ Via Salaria 113, Roma - 00198, Italy \\ \{dipietro, durante, mancini, patil\}@ di.uniroma1.it
}

\begin{abstract}
One-way hash chains have been the preferred choice (over symmetric and asymmetric key cryptography) in security setups where efficiency mattered; despite the ephemeral confidentiality and authentication they assure. They only support forward secrecy and have limitations over the chain size (bounded). In this paper, we show how the use of chameleon functions leads to the generation of practically unbounded one-way chains with constant memory storage requirement, providing forward, and backward secrecy as well. Such a cryptographic tool appears to be a great enabler for a variety of applications that could not be efficiently realized earlier. From our experiments we observed that this new kind of one-way chain formation adds a slight computational burden, which is justifiable by the unique advantages provided under our construction. The basic unit of our construction, chameleon function, can be elegantly used to design trees or even simpler star-like constructs.
\end{abstract}

\section{Introduction}

Entity authentication is one of the core primitives that are required to build dependable and secure systems [5]. Several cryptographic protocols have been proposed (e.g. $[8,9,7])$ to reach this goal. These protocols use cryptographic primitives based on a symmetric key crypto-system or an asymmetric key crypto-system. The appropriateness of a cryptographic protocol for an application depends on the computational costs it incurs to the application. Generally, entity authentication is achieved using asymmetric cryptography and data confidentiality by computationally less expensive symmetric cryptography. But, to realize such

${ }^{*}$ This work was partially funded by the WEB-MINDS project supported by the Italian MIUR under the FIRB program, and by the PRIN 2003 "Web-based Management and representation of Spatial and Geographic Data" program from the Italian MIUR. a mechanism, an underlying security framework is required, called PKI (Public Key Infrastructure). Integration of such a framework with computation-constrained environment or in dynamically changing setups is not practical due to the costs and complexity of the framework.

A widely accepted approach is the use of one-way hash chains [8] (alternatively referred as Lamport chain, in this paper), that provides verifiable data authentication in an efficient way. Much recent work [9], even tries to achieve the characteristics provided by asymmetric cryptography with the help of one-way hash chains and time synchronization between the communicating principals, where ephemeral data confidentiality is provided and backward secrecy is not guaranteed. Though these approaches try to address a particular domain of applications, the philosophy can be extended to much complex scenarios (e.g. group membership, secure multi-cast communication, key renewal etc.), in distributed environment. The issue of key renewal has special significance because of cryptanalytic reasons and hence, for each key renewal the authenticity of the received key must be assured. Lamport chains achieve these objectives efficiently, but with a few limitations: limited chain length and no backward secrecy. Our construction (named "chameleon hash", based on chameleon functions [7, 2, 3, 10]) has done away with these limitations. In comparison with the oneway chain based schemes [6], our solution:

1. allows to generate practically un-bounded one-way chain, whose length is only limited by the finite-ness of the field over which the values are generated,

2. has both storage and computation constant requirement,

3. provides both forward and backward secrecy of communication, and

4. does not require generation of the complete chain before starting its use, since generation and usage of the chain proceed in same direction, where the generation process is at least one step ahead of the later. 


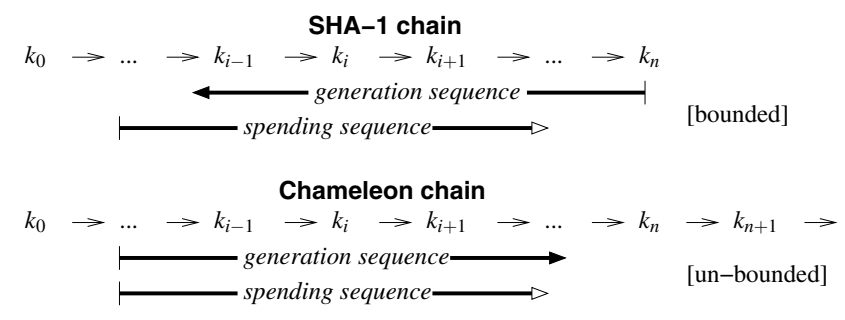

Figure 1. Generation and Spending Sequences in SHA-1 and Chameleon Chains

\section{Background and Overview}

A brief overview of chameleon functions [7, 1, 3, 2] and their properties are given below. To begin with, let us assume that a principal chooses an asymmetric keypair, where $H K_{R}$ denotes the public-key and $C K_{R}$ denotes its corresponding private-key (also called trap-door). Each chameleon hash function is associated with a unique publickey. Let $\mathrm{CH}_{R}(.,$.$) represent the chameleon hash function$ derived from public-key $H K_{R}$. Given a message $m_{i}$ and a random seed $r_{j}$, the $\mathrm{CH}_{R}\left(m_{i}, r_{j}\right)$ function generates a hash value satisfying the following two properties:

- Collision resistance: There is no efficient algorithm that on input the public key $H K_{R}$ can find pairs $m_{i}, r_{i}$ and $m_{z}, r_{z}$ where $m_{i} \neq m_{z}$, such that $\mathrm{CH}_{R}\left(m_{i}, r_{i}\right)=\mathrm{CH}_{R}\left(m_{z}, r_{z}\right)$, except with negligible probability.

- Trapdoor Collision: There is an efficient algorithm that on input the secret key $C K_{R}$, and any pair $m_{i}, r_{i}$, and any additional message $m_{z}$, finds a value $r_{z}$ such that $\mathrm{CH}_{R}\left(m_{i}, r_{i}\right)=\mathrm{CH}_{R}\left(m_{z}, r_{z}\right)$. Therefore,

1. the knowledge of public key $H K_{R}$ allows a user to derive the corresponding chameleon hash function,

2. only the owner of $H K_{R}$ 's corresponding secret key, $C K_{R}$, (also called trapdoor) can efficiently find a collision for any given output;

3. for others, the function $C H_{R}(.,$.$) offers strong$ collision-resistance, i.e., it should be computationally infeasible to find two inputs with the same image under this function.

\section{Construction of Chameleon Chain}

Figure 1 shows how the construction of a SHA-1 based one-way chain differs from chameleon based one. Given a random string $m_{0}$ and a seed $r_{0}$, the server computes $k_{0}$ using $\mathrm{CH}_{R}$. $\quad k_{0}$ (the first session-key) is distributed via a secure channel. On the next re-keying the server generates two random values $m_{1}$ and $r_{1}$, and computes $k_{1}=C H_{R}\left(m_{1}, r_{1}\right)$. Further, leveraging the trapdoor collision property, the server efficiently finds a seed $s_{1}$ such that $\mathrm{CH}_{R}\left(k_{1}, s_{1}\right)=\mathrm{CH}_{R}\left(m_{0}, r_{0}\right)=k_{0}$. Once the client receives $k_{1}$, it can check the authenticity of $k_{1}$ by performing $C H_{R}\left(k_{1}, s_{1}\right)=k_{0}$ check. As need arises, the server can repeat these steps to generate a chain of verifiable keys and use them in the same sequence of their generation. The corresponding steps performed by both parties for generation and verification of session keys are enumerated below;

\section{- Sender/Server :}

1. randomly chooses $m_{i+1}$ and $r_{i+1}$, and creates new key $k_{i+1}=C H_{R}\left(m_{i+1}, r_{i+1}\right)$,

2. chooses $s_{i+1}$ such that; $k_{i}=\mathrm{CH}_{R}\left(m_{i}, r_{i}\right)=\mathrm{CH}_{R}\left(k_{i+1}, s_{i+1}\right)$, and

3. sends the message $p=E_{k_{i}}\left(k_{i+1}, s_{i+1}\right)$ to the user.

\section{- Receiver/Client:}

1. receives the message packet $p$,

2. decrypts the packet $p=E_{k_{i}}^{-1}\left(k_{i+1}, s_{i+1}\right)$ using the old key $k_{i}$, and

3. authenticates $k_{i+1}$ by checking if $k_{i}=C H_{R}\left(k_{i+1}, s_{i+1}\right)$, otherwise discards $k_{i+1}$.

Key generation efficiency: Our construction achieves key authenticity in (memory*computation) complexity $O(1)$ since it requires: 1) on the server side: a) to store the values of the current key $k_{i}$, the next generated key $k_{i+1}$, the public key $H K_{R}$ and the secret key $C K_{R}$; b) to build the next session key $k_{i+1}$ performing the chameleon hash $k_{i+1}=\mathrm{CH}_{R}\left(m_{i+1}, r_{i+1}\right)$ that requires $O\left(\max \left\{\left|m_{i+1}\right|,\left|r_{i+1}\right|\right\}\right)$ modular exponentiations, to find out a chameleon collision $k_{i}=\mathrm{CH}_{R}\left(m_{i}, r_{i}\right)=\mathrm{CH}_{R}\left(k_{i+1}, s_{i+1}\right)$ that requires $O\left(\max \left\{\left|m_{i}\right|,\left|r_{i}\right|\right\}\right)$ modular exponentiations as well, 2) on the client side: a) to store the values of the public key $H K_{R}$, the current key $k_{i}$, and the next generated key $k_{i+1}$ sent by the server; b) the chameleon verification $k_{i}=$ $\mathrm{CH}_{R}\left(k_{i+1}, s_{i+1}\right)$, that requires $O\left(\max \left\{\left|k_{i+1}\right|,\left|s_{i+1}\right|\right\}\right)$ modular exponentiations too. Note that $O\left(\max \left\{\left|k_{i+1}\right|,\left|s_{i+1}\right|\right\}\right)$ is the computational complexity of the schemes proposed in $[7,10]$.

Use of SHA-1 based one-way hash chains as a tool for improving the efficiency of a variety of practical and valuable security applications is well rooted. However, knowing $k_{n}$ and given that the current value in the chain is $k_{i}$, the cost for computing the value $k_{i+1}$ in the hash chain, is a topic that has largely been ignored for long chains. This task may account for a quite noticeable portion of the total computational effort of the protocol. The technique often employed 
is to compute each hash value in the chain (on-the-fly) by iterative applications of the hash function starting from an initial seed. However, such a method has a computational complexity of $O(n-i)$ to generate key $k_{i}$. Another possible method, in which all values are pre-computed and stored, substantially reduces the on-the-fly computational cost, but has a storage complexity of $O(n)$. All straightforward combinations of these two techniques can be shown to have a (memory * computation) complexity of $O(n)$, which can be a substantial computational burden for many resourceconstrained devices, such as wireless sensor networks or hand-held devices.

\section{Advantages of Chameleon Chains}

The ability of the owner of the trap-door to efficiently find collisions gives our construction the following prominent properties.

Practically unbounded one-way chain - Unlike Lamport's one-way chain, our scheme provides a one-way hash chain whose length is restricted by the finiteness of the field the chain is built upon. One-way chains using chameleon functions are always generated and used in same direction (i.e. the generator of the chain is at least one step ahead than the last revealed value), whereas in Lamport's one-way chain the generation and usage proceed in opposite directions (thus limiting the length of the chain).

Backward Secrecy - Knowing a value of any unit from the SHA-1 one-way chain, lets one derive all the values between that point up to the anchor of the chain. This eventually leads to the exposure of all previous communications secured using the hash values of such a chain. Whereas, in our construction, one can derive only the previous value in the chain; not beyond that. This is an important requirement while using one-way chains for managing group memberships (for example). A newly joined/enabled member should not get undue privileges, other than the assigned ones, when the membership action is performed.

Constant memory storage requirement - In one-way chains derived using SHA-1 hash function, the applications follow a trade-off between storage of the actual chain values and/or re-computation of the values. Such a constraint is absent in chameleon chains since it is not necessary to compute the complete chain in advance.

Time synchronization requirement - The applications (e.g. multi-cast) that use Lamport chains need to loosely time-synchronize the communicating ends to establish authenticity of the content distributed. Our scheme does away with this requirement [4].

\section{Conclusions}

In this paper, we have derived an analogy between Lamport's one-way chain and one-way chains based on chameleon functions. Our construction has the following advantages over the former: i) practically unbounded length, ii) backward secrecy, iii) constant memory storage and computation requirement, and iv) no time synchronization requirement. These properties, which are otherwise not realizable, come at an extra computational cost [4].

Finally, note that in this paper we have exposed chaining just as one of the possible construct that we could implement. Chameleon hash functions can be used just as easily to construct trees or even simpler star-like constructs which would eliminate the need for the verifier to have all intermediate values [4]. Chaining is only important if causality of signatures is needed.

Acknowledgement: The authors thank Prof. Gene Tsudik for his valuable suggestions.

\section{References}

[1] G. Ateniese and B. de Medeiros. Identity-based chameleon hash and applications. In Financial Cryptography: 8th International Conference, FC 2004, pages 164-180. Springer LNCS 3110, 2004.

[2] G. Ateniese and B. de Medeiros. On the key exposure problem in chameleon hashes. Cryptology ePrint Archive, Report 2004/243, 2004. http://eprint.iacr.org/.

[3] X. Chen, F. Zhang, and K. Kim. Chameleon hashing without key exposure. In Information Security Conference, pages 87-98. Springer LNCS 3225, 2004.

[4] R. Di Pietro, A. Durante, L. V. Mancini, and V. Patil. Applications of chameleon functions in security domain. Technical Report TR-WEBMINDS-54, CINI, March 2005. http://web-minds.consorzio-cini.it/activities.

[5] R. Di Pietro and L. V. Mancini. Security and privacy issues of handheld and wearable wireless devices. Communications of the ACM, 46(9):75-79, 2003.

[6] N. M. Haller. The S/KEY one-time password system. In Proceedings of the Symposium on Network and Distributed System Security, pages 151-157, 1994.

[7] H. Krawczyk and T. Rabin. Chameleon signatures. In ISOC Network and Distributed System Security Symposium (NDSS), pages 143-154, 2000.

[8] L. Lamport. Password authentification with insecure communication. Commun. ACM, 24(11):770-772, 1981.

[9] A. Perrig, R. Canetti, J. D. Tygar, and D. X. Song. Efficient authentication and signing of multicast streams over lossy channels. In IEEE Symposium on Security and Privacy, pages 56-73, 2000.

[10] A. Shamir and Y. Tauman. Improved online/offline signature schemes. In CRYPTO '01: Proceedings of the 21st Annual International Cryptology Conference on Advances in Cryptology, pages 355-367. Springer LNCS 2139, 2001. 